\title{
Two Bioinspired Methods for Dynamic Signatures Analysis
}

\author{
Jânio Canuto ${ }^{1}$, Bernadette Dorizzi ${ }^{1}$, and Jugurta Montalvão ${ }^{2}$ \\ ${ }^{1}$ Institut Mines-Telecom, Telecom SudParis, CNRS UMR5157 SAMOVAR, \\ Évry, France \\ \{janio.canuto, bernadette.dorizzi\}@telecom-sudparis.eu \\ ${ }^{2}$ Federal University of Sergipe (UFS), Electrical Engineering Department (DEL) \\ São Cristóvão, Sergipe, Brazil \\ jmontalvao@ufs.br
}

\begin{abstract}
This work focuses on the problem of dynamic signature segmentation and representation. A brief review of segmentation techniques for online signatures and movement modelling is provided. Two dynamic signature segmentation/representation methods are proposed. These methods are based on psychophysical evidences that led to the well-known Minimum Jerk Model. These methods are alternatives to the existing techniques and are very simple to implement. Experimental evidence indicates that the Minimum Jerk is in fact a good choice for signature representation amongst the family of quadratic derivative cost functions defined in Section 2.
\end{abstract}

Keywords: Dynamic Signatures, Segmentation, Minimum Jerk.

\section{Introduction}

Handwritten signatures result from voluntary but typically complex gestures of the human hand. As result of their particularities, in most cultures, these graphically recorded gestures have been used for centuries to authenticate documents. Some less straightforward uses of handwritten signatures analysis may include mental illness detection or daily stress measurement.

Beyond potential applications, modelling gestures behind signatures and development of proper ways for identification of basic components (i.e., segments) is a challenging enough matter for scientific research. Segmentation is a crucial step that strongly influences the performance of signature verification systems and, therefore, a special attention has been drawn into this task over the last few decades [1].

This work aims at providing two new signature segmentation methods, based on psychophysiological evidences that led to the development of the well-known Minimum Jerk principle for movement planning. We focus on dynamic signatures, which are represented as a time series of pen-tip position coordinates acquired at a fixed sample rate through the use of specific recording devices, such as tablets, digitizers or smartphones. 
The work is structured as follows: in Section 2 we perform a review of previous works on signature segmentation and movement modelling, with an emphasis on the Minimum Jerk model. In Section 3 we present the proposed algorithms along with some experimental results. Finally, in Section 4, conclusions are drawn.

\section{Previous Works}

\subsection{Signature Segmentation}

Global features (e.g. length, maxima, minima, and mean velocity) are commonly used in signature verification systems but are not very discriminative. Such features can be made local if applied to elements of a segmented signature. Using localized features is sometimes referred to as a stroke-based approach. Although this decomposition has shown to provide good results, it leads to the non-trivial segmentation task [2].

Indeed, signature segmentation is a very complex task due to the high variability between different signatures provided by the same writer. These variations include stretching, compression, omission and addition of parts of the drawing. Segmentation techniques might derive from specific characteristics of handwriting movements or provide segmentations that are well suited for particular verification methods. In [1] we can find a brief review of such methods that are divided in four categories according to which principle they are based on: pen-up/pen-down signals, velocity analysis, perceptual relevant points and dynamic time warping.

For dynamic signatures, a common and very simple segmentation technique uses pressure information for determining writing units, which are determined as the written part between a pen-down and a pen-up movement.

Segmentation techniques based on velocity analysis use different approaches, ranging from simple detection of null [3] velocity to curvilinear velocity signals. The stroke identification step on the Sigma-Lognormal model [4] can also be placed on such category.

A different class of segmentation methods are those based on the detection of perceptually important points. The importance of a point is determined by the rate of change of the writing angle around it. We can also include in this category techniques based on the detection of geometric extremes [5].

In order to allow the segmentation of many signatures into the same number of segments, dynamic time warping (DTW) has been widely used.

Combinations of different techniques can also be found in the literature, in [6] a combination of pressure (first category), velocity (second category) and angle change (third category) is used for segmentation. Further references for dynamic signature segmentations methods can be found in [1].

\subsection{Movement Modelling}

The study of how the central nervous system (CNS) generates and controls the movement has yielded many computational models, some relying on biological neural 
network behaviours, artificial neural networks, equilibrium point hypothesis, coupled oscillators and minimization principles [4].

From a movement planning perspective, the general problem might be posed as follows: It is assumed that human movements are optimally planned according to a latent optimality criterion. Therefore, we need to find what is the optimality criterion used by the CNS. From this formulation, the optimal control theory seems to be the most complete and adequate way for finding an answer [7].

All minimization principles for movement modelling are part of the optimal control approach. Many optimization criteria have been proposed in the literature and can roughly be divided in four categories: kinematic criteria (e.g. minimum jerk), dynamic criteria (e.g. minimum torque-change), muscular and neural criteria (e.g. minimum effort) and energetic criteria (e.g. minimum total work). A detailed review of such criteria can be found in [7] and references therein. Amongst these criteria, Minimum Jerk (MJ) and Minimum Torque-Change (MTC) are the most used in the literature.

Some of these models have already been used for handwriting representation, but only the sigma-lognormal model, which is based on the kinematics theory of rapid human movement, has been used for signature modelling [4]. We, on the other hand, use in our proposed methods the Minimum Jerk principle [8] which to the authors' knowledge has not yet been applied to signature analysis.

Therefore, we now present a more detailed description of the Minimum Jerk model.

The Minimum Jerk Model. This model belongs to the category of the quadratic derivative kinematic criteria, which have a general cost function defined as:

$$
C=\frac{1}{2} \int_{0}^{T}\left(\frac{d^{n} x(t)}{d t^{n}}\right)^{2}+\left(\frac{d^{n} y(t)}{d t^{n}}\right)^{2} d t
$$

where $T$ is the movement duration, $x(t)$ and $y(t)$ are the horizontal and vertical position time series respectively. This class of optimum criteria has as general solution polynomials of order $2 n-1$. The jerk is defined as the third derivative of the movement, therefore $n=3$ and the general solution is a fifth-order polynomial. Other well-known kinematic criteria such as acceleration, snap and crackle can be obtained by setting $n$ equal to 2, 4 and 5 respectively.

In [8] this family of optimum criteria has been studied up to $n=10$, and the authors concluded that the Minimum Jerk is the most suitable for human movement modelling. They were able to properly reproduce the two-thirds power law using the minimum jerk. Using the peak to average velocity ratio as a single scalar projection of velocity profiles, they also found that the $\mathrm{MJ}$ is the best suited criterion. Indeed, previous experimental evidences showed that this ratio is about 1.8 (with $10 \%$ standard deviation) for reaching movements and the MJ yields a ratio of 1.875 for this class of movements.

Some works point that the Minimum Jerk model is unable to produce asymmetric velocity profiles [7, 9], however this is only true if velocity and acceleration at both the beginning and the end of the movement are null, which is not a requirement of the model itself. Furthermore, in [7] it is noted that MJ predictions are not in agreement with experimental data when the movement occurs on the vertical plane or when the 
target is not a single point but an infinite set of point (a straight line, for instance); however MJ predictions work properly on the horizontal plane for point-to-point movements. These conditions can both be assumed for a signing movement.

\section{Proposed Method}

All experiments described in this section have been performed over the MCYT-100 Database [10], which consists of 25 genuine and 25 forged signatures from 100 different writers. These signatures have been acquired with a Wacom® Tablet at a fixed sample rate of $100 \mathrm{~Hz}$. In our experiments, since we are focused on segmentation and representation, and not on verification, only the genuine subset has been used.

Our first segmentation method is based on the peak to mean velocity ratio observed on the psychophysical experiments realized during the developments of the MJ principle. We sequentially search for signature segments that comply with the expected 1.8 ratio.

In other words, we segment the signature as a series of point-to-point reaching movements. This method can be categorized amongst the velocity analysis segment techniques. For a given signature, the algorithm can be described as follows:

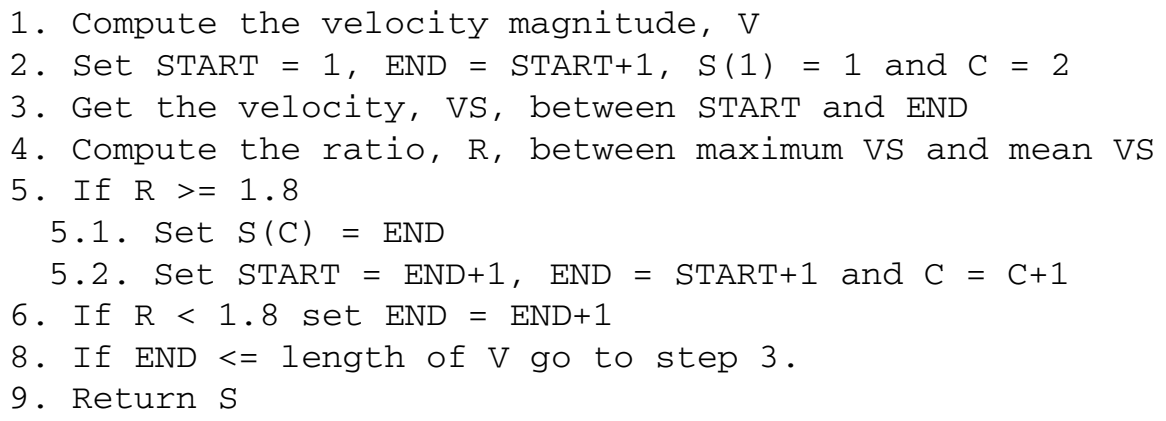

This extremely simple procedure provides a stable segmentation amongst different signatures of the same writer, with an average coefficient of variation of $13 \%$ for all writers. In Figure 1 are shown some examples of segmentation for five different signatures of two writers on the MCYT-100 Database.

It is interesting noting that using this method we are able to remove small artefacts that are often present at the beginning and end of the acquired signatures. The obtained segmentations roughly correspond to changes in the dynamic behaviour (e.g. loops, waves and straight lines).

Visual inspection of the obtained segmentation seems to yield "natural" segments. This technique has the advantages of not being tied to any verification technique, and can be used for any signature analysis task. Furthermore, the 1.8 threshold used for segmentation is based on sound psychophysical experiments that have been reproduced many times over the last 30 years.

This segmentation does not provide a model for the resulting elements, as those in the Sigma-Lognormal model. One could use the result of the MJ principle (i.e. a fifth 
order polynomial) in order to model each of the resulting segments, however not all of the elements can be properly modelled by such simple functions, resulting in a very poor reconstruction quality, especially on signatures containing many consecutive loops.
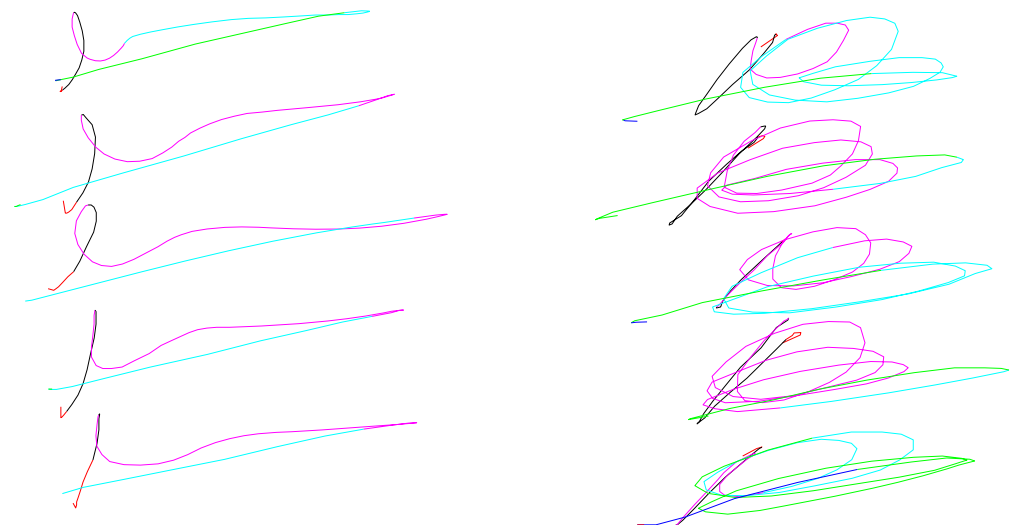

Fig. 1. Segmentation of five different signatures of two writers using the velocity ratio criterion

The reconstruction quality is measured through the Velocity SNR, as in [4], defined as:

$$
S N R_{v}=10 \log \left(\frac{\int_{0}^{T}\left[v_{x}^{2}(t)+v_{y}^{2}\right] d t}{\int_{0}^{T}\left[\left(v_{x}(t)-v_{x x}(t)\right)^{2}+\left(v_{y}(t)-v_{y y}(t)\right)^{2}\right] d t}\right)
$$

where $v_{x}(t)$ and $v_{y}(t)$ are respectively the horizontal and vertical velocities on the original signature, and $v_{x x}(t)$ and $v_{y y}(t)$ are the horizontal and vertical velocities of the reconstructed signature, respectively. For the abovementioned representation an average $S N R_{V}$ of $10.7 \mathrm{~dB}$ is obtained for the MCYT-100 database.

We now propose a second method, based on the MJ Model itself, that result in a piecewise polynomial representation of the signature. This approach models each "writing element" as a fifth order polynomial. The procedure consists in sequentially finding the longer segments that can be adequately represented (according to a given reconstruction quality threshold) by a fifth order polynomial [11].

Notice that the 1.8 peak to mean velocity ratio is only observed on reaching movements, in which starting and ending velocity and acceleration are considered to be null (thus, resulting in a symmetric velocity profile), different boundary conditions on the MJ Model lead to different velocity ratios and velocity profiles. Therefore, fitting the fifth-order polynomial to the signature data allows for a better representation of the velocity profiles and can provide not only segmentation but an adequate 
representation for each segment. The algorithm for this method can be described as follows:

1. Normalize the trajectory through a min-max procedure

2. Set $L=1$

3. Set $R=L+5$

4. Fit a $5^{\text {th }}$ order polynomial to the points in the interval $[X(L): X(R)]$

5. Calculate the Velocity SNR for the interval

6. If SNR $>=$ Threshold

6.1. Set $R=R+1$ and go to step 4 .

7. If SNR < Threshold

7.1. Set $L=R-1$ and go to step 3 .

8. If $R>$ length of $X$, stop.

9. Return the list of L values.

This procedure is still very simple but much more computationally intensive than the previous one because of the fitting procedure. We chose not to use the Jerk value itself because the numerical estimation of third order derivatives from time-sampled data leads to large numerical errors. We now have a piecewise polynomial representation of signing movements that can be properly predicted by the MJ model.

One of the advantages of such procedure is that the representation quality can be chosen by the user to fit his/her needs. In Figure 2 we present the segmentation obtained for the same signatures shown in Figure 1 with a SNR $R_{v}$ of $15 \mathrm{~dB}$.
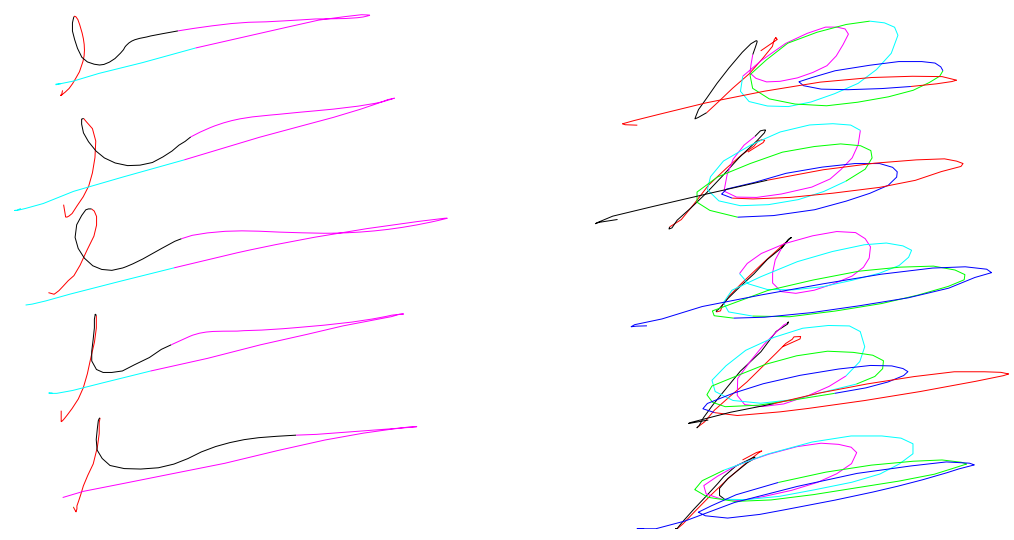

Fig. 2. Segmentation of five different signatures of two writers using the MJ criterion at $15 \mathrm{~dB}$ SNR

Even though for the signatures on the right column in Figure 2 we observe more segments than using the first method, on average, for the whole database this procedure produce fewer segments than the previous one. Furthermore, the number of 
segments obtained for each writer is more stable than before, with a coefficient of variation of $7.3 \%$, that is even lower than the variability for the lengths of the signatures which is of $8 \%$.

Now the obtained signature elements are basically arcs and single loops, most of them containing at least one inflection point. Along with the automatic segmentation we have an alternative representation for the signature as a sequence of fifth order polynomials. Considering the trade-off between compression rate and reconstruction quality, we did experiments similar to those in [8] with different cost functions belonging to the family defined by Equation (1), with $n$ ranging from one to five. Once again the Minimum Jerk $(n=3)$ seems to be the best option, acting as a limit to the compression-quality trade-off. For a SNR of $15 \mathrm{~dB}$ all of the higher order $(n \geq 3)$ solutions attain the same compression rate of $73.3 \%$. In Figure 3 we show the compression-quality trade-off curves for each different cost function.

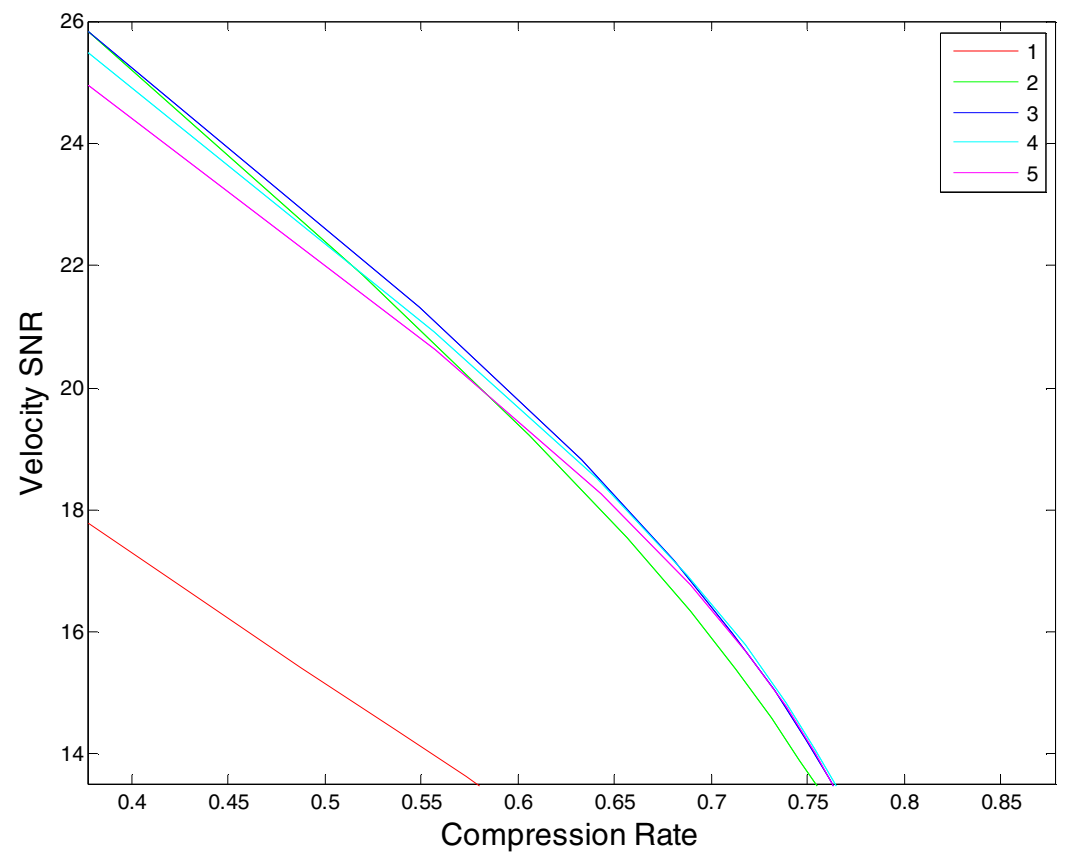

Fig. 3. Representation quality and compression rate tradeoff for minimum velocity $(n=1)$, acceleration $(n=2)$, jerk $(n=3)$, snap $(n=4)$ and crackle $(n=5)$

\section{Conclusions and Future Work}

Two methods for automatic signature segmentation and one for signature representation have been presented. These methods are based on the Minimum Jerk Principle and to the authors knowledge it is the first use of such principle for online signatures. 
Both methods are very easy to implement and can be used as alternatives to existing methods.

As pointed out before, it is hard to determine if our approach is better than existing methods because such evaluation strongly depends on what the segmentation/representation is going to be used to. An advantage of the proposed model is the possibility of choosing the desired representation error.

The proposed method can be understood as an alternative use of the Minimum Jerk criterion as a basis function for a piecewise representation. In such way the commonly used velocity and acceleration constraints are not needed as well as any manually inserted via-points.

We believe that the ratio between the number of segments obtained by such method and the original length of the signature may be used as a "complexity" measure in a similar fashion to the Normalized Lempel-Ziv Complexity [12], we have an ongoing work on the demonstration of this link between detected segments and LempelZiv Complexity.

The next step in our work consists in integrating the proposed methods in a recognition system in order to obtain a higher level assessment of its impact. We intend to analyze two main aspects: the reduction of the computational cost due to the use of a more compact representation and the effect of such model in the system performance.

Furthermore, since the Minimum Jerk Model is derived from the analysis of healthy individuals' movements, this complexity measure may be used in the early diagnosis of motor diseases such as Parkinson's and Dyskinesia.

\section{References}

1. Impedovo, D., Pirlo, G.: Automatic Signature Verification: The State of the Art. IEEE Trans. Syst., Man, and Cybern. - Part C: App. and Rev. 38(5), 609-635 (2008)

2. Yue, K.W., Wijesoma, W.S.: Improved Segmentation and Segment Association for Online Signature Verification. In: Proc. IEEE Int. Conf. Syst., Man, Cybern., vol. 4, pp. 2752-2756 (2000)

3. Dolfing, J.G.A., Aarts, E.H.L., van Oosterhout, J.J.G.M.: On-line Signature Verification with Hidden Markov Models. In: Proc. 4th Int. Conf. Pat. Rec., vol. 2, pp. 1309-1312 (1998)

4. Plamondon, R., O’Reilly, C., Galbally, J., Almaksour, A., Anquetil, E.: Recent developments in the study of rapid human movements with the kinematic theory: Applications to handwriting and signature synthesis. Pat. Rec. Letters (June 15, 2012) (in press)

5. Lee, J., Yoon, H.-S., Soh, J., Chun, B.T., Chung, Y.K.: Using geometric extrema for segment-to-segment characteristics comparison in online signature verification. Pat. Rec. 37, 93-103 (2004)

6. Qu, T., Saddik, A.E., Adler, A.: A stroke based algorithm for dynamic signature verification. In: Proc. Can. Conf. Ele. Comp. Eng. (CCECE), pp. 461-464 (2004)

7. Berret, B.: Integration de la force gravitaire dans la planification motrice et le controle des mouvements du bras et du corps. PhD Thesis, Pozzo, T., Gauthier, J.-P. (advisors), Bougogne Univesity (2008) 
8. Richardson, M.J.E., Flash, T.: Comparing Smooth Arm Movements with the Two-Thirds Power Law and the Related Segmented-Control Hypothesis. J. Neuroscience 22(18), 8201-8211 (2002)

9. Dijoua, M., Plamondon, R.: The Limit Profile of a Rapid Movement Velocity. Human Movement Science 29(1), 48-61 (2010)

10. Ortega-Garcia, J., Fierrez-Aguilar, J., Simon, D., Gonzalez, J., Faundez-Zanuv, M., Espinosa, V., Satue, A., Hermanez, I., Igarza, J.J., Vivaracho, C., Escudero, D., Moro, Q.I.: MCYT baseline corpus: a bimodal biometric database. IEEE Proc. Vis., Im., Sig. Proc. 150(6), 395-401 (2003)

11. Canuto, J., Dorizzi, B., Montalvão, J.: Dynamic Signatures Representation Using the Minimum Jerk Principle. In: Proc. 4th IEEE Biosig. Biorob. Conf. (ISSNIP), pp. 1-6 (2013)

12. Ziv, J.: Coding Theorems for Individual Sequences. IEEE Trans. Inf. Theory IT-24(4), 405-412 (1978) 